\title{
A Re-Interpretation of the Concept of Nash Equilibrium Based on the Notion of Social Institutions*
}

\author{
Guilherme Carmona \\ Universidade Nova de Lisboa
}

January 29, 2003

\begin{abstract}
We define social institutions as strategies in some repeated game. With this interpretation in mind, we consider the impact of introducing requirements on strategies which have been viewed as necessary properties for any social institution to endure. The properties we study are finite complexity, symmetry, global stability, and semiperfection. We show that: (1) If a strategy satisfies these properties then players play a Nash equilibrium of the stage game in every period; (2) The set of finitely complex, symmetric, globally stable, semi-perfect equilibrium payoffs in the repeated game equals the set of Nash equilibria payoffs in the stage game; and (3) A strategy vector satisfies these properties in a Pareto optimal way if and only if players play some Pareto optimal Nash equilibrium of the stage game in every stage. These results provide a social institution interpretation of Nash equilibrium: individual behavior in enduring social institutions is described by Nash equilibria.
\end{abstract}

*This paper is a revised version of Chapter 3 of my Ph.D. thesis. I wish to thank my advisors, Narayana Kocherlakota, and Marcel K. Richter, for their guidance, encouragement, and very helpful comments and suggestions. I wish also to thank Kemal Badur, Larry Blume, Nuno Garoupa, Leonid Hurwicz, Max Jodeit, Andy McLennan and participants at the Game 2002, and Asset 2002 conferences for very helpful comments. While they all improved the material in many ways, all remaining errors are mine. Financial support from the Subprograma Ciência e Tecnologia do $2^{\circ}$ Quadro Comunitário de Apoio is greatufully acknowledged. 


\section{Introduction}

The concept of Nash equilibrium is one of the central concepts in economic analysis. Notwithstanding, many authors have proposed alternative notions of equilibria by arguing that in certain circumstances some Nash equilibria may be implausible. ${ }^{1}$ These refinements inevitably lead to the question: When is it appropriate to apply the concept of Nash equilibrium?

In his Ph.D. dissertation, John Nash proposed two interpretations of his equilibrium concept, with the objective of showing how equilibrium points "(...) can be connected with observable phenomenon." (Nash (1950, p. 21)) One interpretation is rationalistic: if we assume that players are rational, they know the full structure of the game, the game is played just once, and there is just one Nash equilibrium, then players will play according to that equilibrium. $^{2}$

A second interpretation, which Nash names mass-action interpretation, is less demanding on the players. In this interpretation, "[i] t is unnecessary to assume that the participants have full knowledge of the total structure of the game, or the ability and inclination to go through any complex reasoning processes." (Nash (1950, p. 21)) What is assumed is that there is a population of participants for each position in the game, which will be played throughout time by participants drawn at random from the different populations. If there is a stable average frequency with which each pure strategy is employed by the "average member" of the appropriate population, then this stable average frequency constitutes a Nash equilibrium.

The framework developed by Nash (1950) for the mass-action interpretation is also appealing as a description of individuals' interaction in large societies. In particular, Okuno-Fujiwara and Postlewaite (1995), and Kandori (1992) have used a similar framework to stress the importance of social institutions in individual decision-making: In environments in which individuals interact throughout time, and have limited information about the others, social institutions can be helpful to summarize the past, and form expectations about the behavior of other players. Will social institutions typically recommend players to play Nash equilibria? The main objective of this paper is to argue that in those social institutions that are likely to endure, players play a Nash equilibrium in every period, and hence provide

\footnotetext{
${ }^{1}$ See van Damme (1991) for a summary of the literature on refinements of Nash equilibria.

${ }^{2}$ For a formal discussion of these ideas, see Aumann and Brandenburger (1995).
} 
a social institution interpretation of Nash equilibrium.

In this paper, we define social institutions as strategies in some repeated game. ${ }^{3}$ Although there may not be a complete agreement on the notion of social institutions, several authors have described them in this fashion. For example, Schotter (1981, p. 24) argues that “(..) social institutions can be best described as noncooperative equilibria of supergames that involve repeated play of some particular constituent game (...)."

The case for interpreting social institutions as strategies can be further strengthened by the fact that each strategy in any repeated game can be represented as an automaton (see Kalai and Stanford (1988, Lemma 3.1, p. 401).) An automaton is described by a set of states (one of which is specified to be the initial state), by a transition function (which gives the next round's state as a function of the current round's state and actions), and by a behavior function (which prescribes behavior according to the state of the automaton). If we interpret the states of the automaton as "social roles," then an automaton (i.e., a strategy) prescribes players' behavior according to their social role, which is in turn determined by their past actions. This interpretation corresponds closely, for example, to Roberts and Holdren (1972, p. 110)'s definition of a social institution:

"An institution will be defined as a system of rules applicable to established practices (or situations) and generally accepted by the members of a social system. (...) The essential point is that an institution specifies consequences of individual or group action which can be expected. Given an existing institution, an individual or a group knows to some extent the reaction its activities will evoke."

Also, it corresponds closely to Okuno-Fujiwara and Postlewaite (1995, p. $80)$ 's definition of a social norm:

"A norm for a society will provide what we call a "social standard of behavior" that will prescribe to a player in a conflict situation with another player a particular action that depends on his characteristics and those of his opponent. We will use the term status to describe an individual's relevant characteristics. A social standard of behavior will thus prescribe an agent's action as a function of his and his opponent's status."

\footnotetext{
${ }^{3}$ For more on the notion of social institutions, see Carmona (2002c).
} 
Viewing social institutions as supergame strategies, several authors have argued that equilibria in such games should satisfy certain additional properties. These additional properties should be imposed not only because they are regarded as desirable from a normative point of view, but also, because they are viewed as properties that social institutions typically have, and which may be necessary for any social institution to endure. Typically, those properties fall in one of the following categories:

1. simplicity,

2. symmetry,

3. stability,

4. complexity.

Aumann (1981, p. 21) proposed the use of finite automata in order to define a simple class of repeated game strategies. ${ }^{4}$ That social institutions should be simple, and represented by finite automata was defended, or at least assumed, by Kandori (1992, p. 72), Okuno-Fujiwara and Postlewaite (1995, p. 83), Rubinstein (1986, p. 84), and Schotter (1981, p. 57).

Symmetry is a property that is often assumed to capture the notion of a common culture or a common legal system. We will call a strategy symmetric if all players share a common state space, and a common transition function, properties which were assumed in Okuno-Fujiwara and Postlewaite (1995)'s social norms.

One stability property that has been advocated is that mistakes that players might make in the beginning of the game shouldn't have a longterm effect in players' payoffs - this property was named global stability by Kandori (1992, p. 73). Some other stability properties that are close in spirit to global stability were proposed by Okuno-Fujiwara and Postlewaite (1995, p. 100).

It has been argued earlier that players should use strategies that can be represented by finite automata. Hence, in this case, it is natural to define the complexity of a strategy by the size (i.e., the number of states) of the smallest automata that implements it (see Kalai and Stanford (1988, Theorem

\footnotetext{
${ }^{4}$ Finite automata were introduced by McCulloch and Pitts (1943); see also Hopcroft and Ullman (1979).
} 
3.1, p. 401). $)^{5}$ Regarding complexity considerations, it has been advocated that players' preferences should be assumed to depend both on repeated game payoffs and the complexity of the strategies they use. One equilibrium concept defined in terms of such preferences is Rubinstein (1986, p. 90)'s semi-perfection.

We study the structure of finitely complex, symmetric, globally stable, semi-perfect equilibria. Our main results are:

1. A strategy vector is a finitely complex, symmetric, globally stable, semiperfect equilibrium only if players play a Nash equilibrium of the stage game in every period.

2. The set of finitely complex, symmetric, globally stable, semi-perfect equilibrium payoffs in the repeated game equals the set of Nash equilibria payoffs in the stage game, and

3. A strategy vector is a Pareto optimal, globally stable, symmetric, finitely complex, semi-perfect equilibrium if and only if players play some Pareto optimal Nash equilibrium of the stage game in every stage.

These results provide a social institution interpretation of Nash equilibrium: In a given society, simple, symmetric, stable and minimally complex behavior associated with social institutions is described by Nash equilibria.

\section{$2 \quad$ Notation and Definitions}

Our notation follows closely the one used by Kalai and Stanford (1988). A normal form game $G$ is defined by

$$
G=\left(N,\left(A_{i}\right)_{i \in N},\left(u_{i}\right)_{i \in N}\right)
$$

where: (1) $N$ is the set of players, (2) $A_{i}$ is a the set of player $i$ 's actions, and (3) for all $i \in N, u_{i}: A \rightarrow \mathbb{R}$, where $A=\prod_{i \in N} A_{i}$, is player $i$ 's payoff function. We assume that $N$ is a finite set, and that $N=\{1, \ldots, n\}$. The set $A_{i}, i \in N$, may or may not be finite.

\footnotetext{
${ }^{5}$ Alternative definitions of the complexity of a strategy are proposed in Banks and Sundaram (1990), and in Lipman and Srivastava (1990).
} 
The supergame of $G$ consists of an infinite sequence of repetitions of $G$ taking place in periods $t=1,2,3, \ldots$ At period $t$ the players make simultaneous moves denoted by $a_{i}^{t} \in A_{i}$ and then each player learns his opponent's move.

Finally we need to specify the strategies players can use and also a way to evaluate payoff in the supergame of $G$. For $k \geq 1$, a $k$-stage history is a $k$-length sequence $h_{k}=\left(a_{1}, \ldots, a_{k}\right)$, where, for all $1 \leq t \leq k, a_{t} \in A$; the space of all $k$-stage histories is $H_{k}$, i.e., $H_{k}=A^{k}$ (the $k$-fold Cartesian product of $A$.) The notation $e$ stands for the unique 0 -stage history - it is a 0 -length history that represents the beginning of the supergame. The set of all histories is defined by $H=\bigcup_{n=0}^{\infty} H_{n}$.

For every $h \in H$, define $h^{r} \in A$ to be the projection of $h$ onto its $r^{\text {th }}$ coordinate. For every $h \in H$ we let $\ell(h)$ denote the length of $h$. For two positive length histories $h$ and $\bar{h}$ in $H$ we define the concatenation of $h$ and $\bar{h}$, in that order, to be the history $(h \cdot \bar{h})$ of length $\ell(h)+\ell(\bar{h}):(h \cdot \bar{h})=$ $\left(h^{1}, h^{2}, \ldots, h^{\ell(h)}, \bar{h}^{1}, \bar{h}^{2}, \ldots, \bar{h}^{\ell(\bar{h})}\right)$. We also make the convention that $e \cdot h=$ $h \cdot e=h$ for every $h \in H$.

It is assumed that at stage $k$ each player knows $h_{k}$, that is, each player knows the actions that were played in all previous stages. As for strategies, players choose behavioral strategies. That is, in each stage $k$, they choose a function from $H_{k-1}$ to $A_{i}$ denoted $f_{k}^{i}$, for player $i \in N$. The set of player $i$ 's strategies is denoted by $\Sigma_{i}$, and $\Sigma=\prod_{i \in N} \Sigma_{i}$ is the joint strategy space. Finally, a strategy vector is $f=\left(\left\{f_{k}^{i}\right\}_{k=1}^{\infty}\right)_{i \in N}$.

Any strategy $f \in F$ induces an history $h(f)$ as follows:

$$
h^{1}(f)=f(e), \quad h^{k}(f)=f\left(h^{1}(f), \ldots, h^{k-1}(f)\right),
$$

for $k \in \mathbb{N}$.

The payoff in the supergame of $G$ is, for $\delta \in(0,1)$, the discounted sum of stage game payoffs:

$$
U^{i}(f)=(1-\delta) \sum_{k=1}^{\infty} \delta^{k-1} u^{i}\left(h^{k}(f)\right) .
$$

Given an individual strategy $f_{i} \in \Sigma_{i}$ and a history $h \in H$ we denote the individual strategy induced by $f_{i}$ at $h$ by $f_{i} \mid h$. This strategy is defined pointwise on $H$ : $\left(f_{i} \mid h\right)(\bar{h})=f_{i}(h \cdot \bar{h})$, for every $\bar{h} \in H$. We will use $(f \mid h)$ to denote $\left(f_{1}\left|h, \ldots, f_{n}\right| h\right)$ for every $f \in S$ and $h \in H$. We let $\Sigma_{i}\left(f_{i}\right)=\left\{f_{i} \mid h\right.$ : $h \in H\}$ and $\Sigma(f)=\{f \mid h: h \in H\}$. 


\subsection{Automata and Symmetry}

For $i \in N$, let $f_{i}$ be a strategy for player $i$. We define an equivalence relation on $H$, by declaring the histories $h$ and $\bar{h}$ equivalent relative to $f_{i}, h \sim_{f_{i}} \bar{h}$, if for every history $\beta \in H, f_{i}(h \cdot \beta)=f_{i}(\bar{h} \cdot \beta)$. In words, the behavior prescribed by $f_{i}$ after $h$ is the same as the behavior prescribed by $f_{i}$ after $\bar{h}$. Let $H / \sim_{f_{i}}$ denote the quotient set of $H$ relative to $\sim_{f_{i}}$. Then the complexity of $f_{i}$, denoted by $\operatorname{comp}\left(f_{i}\right)$, is defined to be the cardinality of $H / \sim_{f_{i}}$ (see Kalai (1990, Theorem 4.2, p. 144).) Let $F_{i}$ denote the set of player $i$ 's strategies with finite complexity, and let $F=\prod_{i \in N} F_{i}$. Also, let $[h]_{f_{i}}$ denote the equivalence class of $h \in H$; when its clear which equivalence relation we are refereing to, we write $[h]$ for $[h]_{f_{i}}$.

An automaton for player $i$ is a triple $I_{i}=\left(\left(S_{i}, s_{i}^{0}\right), T_{i}, B_{i}\right)$ where: $S_{i}$ is a set of states; $s_{i}^{0}$ is an initial state; $T_{i}: S_{i} \times A \rightarrow S_{i}$ is a transition function; and $B_{i}: S_{i} \rightarrow A_{i}$ is a behavior function. Any automaton $I_{i}$ for player $i$ induces a strategy $f_{I_{i}}$ as follows: for an history $h \in H$ with $\ell(h)=m$ define inductively

$$
s_{i}^{0}(h)=s_{i}^{0}, \quad s_{i}^{r}(h)=T_{i}\left(s_{i}^{r-1}(h), h^{r}\right) \quad \text { for } \quad r=1,2, \ldots, m,
$$

and then $f_{I_{i}}(h)=B_{i}\left(s^{m}(h)\right)$.

Given a strategy $f_{i} \in F_{i}$ for player $i$, we say that the automaton $I_{i}$ implements $f_{i}$ if $f_{i}=f_{I_{i}}$. For $f_{i} \in F_{i}$ we see that $I_{f_{i}}$ defined by $S_{f_{i}}=H / \sim_{f_{i}}$, $s_{f_{i}}^{0}=[e], T_{f_{i}}([h], a)=[h \cdot a]$ and $B_{f_{i}}([h])=f_{i}(h)$, implements $f_{i}$. Moreover, $I_{f_{i}}$ is minimal in the sense that if $I_{i}$ also implements $f_{i}$ then the cardinality of $S_{f_{i}}$ is less than or equal to the cardinality of $S_{i}$ (see Kalai (1990, Theorem 4.2 , p. 144), and also Kalai and Stanford (1988, Theorem 3.1, p. 401).) Given this result, we will regard any strategy as an automaton.

The following notation will be useful: Given a strategy $f \in \Sigma$ and a state $s \in S=\prod_{i \in N} S_{i}$ we denote the strategy induced by $f$ at $s$ by $f \mid s$. The strategy $f \mid s$ is just like $f$, except perhaps on the initial state: for any $i \in N$, if $f_{i}=\left(\left(S_{i}, s_{i}^{0}\right), T_{i}, B_{i}\right)$, then $f_{i} \mid s=\left(\left(S_{i}, s_{i}\right), T_{i}, B_{i}\right)$.

We will say that a strategy is symmetric if players condition their play on a common state variable, and this common state variable evolves according to a common transition function.

Definition 1 A strategy vector $f$ is symmetric if for all $i, j \in N, S_{i}=S_{j}$, $s_{i}^{0}=s_{j}^{0}$, and $T_{i}=T_{j}$.

We interpret symmetry as a common culture, or a common legal system. 


\section{$2.2 \quad$ Stability}

In this section, we give the stability definition for repeated game strategies that we will use.

Definition 2 (Kandori (1992)) Let $f \in \Sigma$ and let $\left(\bar{u}_{i}\right)_{i \in N}=\left(U^{i}(f)\right)_{i \in N}$. Then $f$ is globally stable if for all $i \in N$, and $s \in S$,

$$
\lim _{k \rightarrow \infty} U_{k}^{i}(f \mid s)=\bar{u}_{i}
$$

where $U_{k}^{i}(f \mid s)=(1-\delta) \sum_{t=k}^{\infty} \delta^{t-k} u_{i}\left((f \mid s)_{t}\right)$ is player $i$ 's continuation payoff at stage $k$.

Note that in the definition of global stability the state $s$ is any possible state, not necessarily the one implied by the play of the equilibrium strategy. The requirement of global stability is that no matter what the past has been, future play of the equilibrium strategies will asymptotically give players the same payoff as if the equilibrium strategies were always followed. Kandori introduced this concept in the context of his work on social norms to capture the intuition that social norms are generally "robust to the mistakes of players" and that they allow "players to test various actions in order to learn [them]".

\subsection{Complexity}

As Rubinstein (1998, p. 137) writes, "[a]t the heart of our discussion in this [section] is the trade-off often facing a decision maker when choosing a strategy. On one hand, he hopes his strategy will serve his goals; on the other hand, he would like it to be as simple as possible. There are many reasons why a player may value simplicity: a more complex plan of action is more likely to break down, is more difficult to learn and may require more time to be implemented. We will not examine these reasons here but simply assume that complexity is costly and under the control of the player."

We assume as in Abreu and Rubinstein (1988, p. 1264) that players' preferences depend both on repeated game payoffs and the complexity of the strategies they use. For any player $i \in N$, let $\succeq_{i}$ be player $i$ 's preference relation on $\mathbb{R} \times \mathbb{N}$. We assume that $\succeq_{i}$

1. is increasing in the payoff:

$$
a>b \text { and } \alpha=\beta \text { implies }(a, \alpha) \succ_{i}(b, \beta) .
$$


2. is decreasing in complexity:

$$
a=b \text { and } \alpha>\beta \text { implies }(a, \alpha) \prec_{i}(b, \beta) \text {. }
$$

An equilibrium concept defined using the above preference relation is:

Definition 3 (Rubinstein (1986)) A strategy vector $f \in F$ is a semiperfect equilibrium if for all $i \in N, s \in S_{1} \times \cdots \times S_{n}$ and $g_{i} \in F_{i}$,

$$
\left(U^{i}(f \mid s), \operatorname{comp}\left(f_{i}\right)\right) \succeq_{i}\left(U^{i}\left(\left(g_{i}, f_{-i}\right) \mid\left(s_{i}^{g 0}, s_{-i}\right)\right), \operatorname{comp}\left(g_{i}\right)\right) .
$$

A particular case, considered in Rubinstein (1986), arises when players have lexicographic preferences over payoffs and complexity. This preference relation, denoted by $\succeq_{L}$ is defined as follows: for $(a, \alpha),(b, \beta)$ in $\mathbb{R} \times \mathbb{N}$, $(a, \alpha) \succ_{L}(b, \beta)$ if and only if $a>b$ or $a=b$ and $\alpha>\beta$. It is then clear that if players have lexicographic preference, then any semi-perfect equilibrium is a subgame prefect equilibrium. This result is not true for general preferences in games with more than two players. However, for two player games with preferences satisfying properties 1 and 2 above, it is true that any semi-perfect equilibrium is a subgame prefect equilibrium (see Piccione (1992, Theorem 2, p. 187)).

\section{$3 \quad$ Structure of Equilibria}

In this section, we state and prove our main results, concerning the structure of equilibria. They provide a characterization of the (finitely complex, symmetric, globally stable, semi-perfect) equilibrium payoffs, and of the Pareto optimal equilibria. Also, they provide necessary conditions on the form of equilibrium strategies.

Our first result shows that all states will be used in equilibrium, and that any state can be reached starting from any other state. Note that any symmetric, finitely complex strategy $f$ induces a Markov Chain on the common state space $S=S_{1}=\cdots=S_{n}$. If $\Pi$ denotes its transition matrix, it follows that $\pi_{s \bar{s}}$ equals either 1 or 0 , for all $s, \bar{s} \in S$ - that is, $f$ induces a deterministic Markov Chain on $S$. Formally, the Markov chain is determined by the following relation:

$$
\pi_{s \bar{s}}=1 \text { if and only if } T(s, f(s))=\bar{s}
$$


Lemma 1 Let $f \in F$ be a finitely complex, symmetric, semi-perfect equilibrium, and $\Pi$ be the transition matrix of the Markov chain induced on $S$ by $f$. Then, $\Pi$ is irreducible.

Proof. We will show that all states $s \in S$ communicate, that is, for all $s$ and $\bar{s} \in S$, there is $k \in \mathbb{N}$ such that $\pi_{s \bar{s}}^{(k)}>0$.

Note that it is enough to show that for all $s$ and $\bar{s} \in S$ such that $s \neq \bar{s}$, there is $k \in \mathbb{N}$ such that $\pi_{s \bar{s}}^{(k)}>0$. This follows because if $k$ is such that $\pi_{s \bar{s}}^{(k)}>0$ and $\bar{k}$ is such that $\pi_{\bar{s} s}^{(\bar{k})}>0$, then $\pi_{s s}^{(k+\bar{k})} \geq \pi_{s \bar{s}}^{(k)} \pi_{\bar{s} s}^{(\bar{k})}>0$.

Let $s$ and $\bar{s} \in S, s \neq \bar{s}$, and suppose that for all $k \in \mathbb{N}$ we have $\pi_{s \bar{s}}^{(k)}=0$. Then any player would deviate from $f$ at state $s$, since he could remove the state $\bar{s}$ and still obtain the same payoff.

More formally, define an automaton $\tilde{f}_{1}$ as follows: $\tilde{S}_{1}=S \backslash \bar{s}, s_{1}^{0}=s$,

$$
\tilde{T}_{1}(\tilde{s}, a)= \begin{cases}s & \text { if } T(\tilde{h}, a)=\bar{s} \\ T(\tilde{h}, a) & \text { otherwise }\end{cases}
$$

and $\tilde{B}_{1}(\tilde{s})=B_{i}(\tilde{s})$. Since $\pi_{s \bar{s}}^{(k)}=0$ for all $k \in \mathbb{N}$, it follows that $\left(\tilde{f}_{1}, f_{-1} \mid s\right)$ gives the same payoff to player 1 as $f \mid s \operatorname{but} \operatorname{comp}\left(\tilde{f}_{1}\right)<\operatorname{comp}\left(f_{1}\right)$. Hence, it follows that $f$ is not a semi-perfect equilibrium, and the lemma follows by contraposition.

Lemma 1 implies that we can order $S$, and thus write $S=\left\{s_{0}, s_{1}, \ldots, s_{L}\right\}$, with $L=|S|-1$, in such a way that $s_{k}=T\left(s_{k-1}, B\left(s_{k-1}\right)\right)$, for all $k=$ $1, \ldots, L$, and $s_{0}=T\left(s_{L}, B\left(s_{L}\right)\right)$. In other words, the play of the game will induce a cycle on the set of states starting from the first period.

Since the states that are used in the play of the game will cycle, so will the continuation payoffs for any given player. However, global stability requires that continuation payoffs converge, which is possible only if they are all equal. This is the content of the following lemma.

Lemma 2 Let $f \in F$ be a finitely complex, symmetric, globally stable, semiperfect equilibrium, and let $\left(\bar{u}_{i}\right)_{i \in N}=\left(U^{i}(f)\right)_{i \in N}$.

Then for all $i \in N$ and $h \in H$,

$$
U^{i}(f \mid s)=\bar{u}_{i}
$$

Furthermore, $u_{i}(B(s))=\bar{u}_{i}$. 
Proof. Let $i \in N$ and $s \in S$. Since $S=\left\{s_{0}, s_{1}, \ldots, s_{L}\right\}$, with $L=|S|-1$, then $s=s_{n}$ for some $0 \leq n \leq L$. We have then, for all $k \in \mathbb{N}$,

$$
U_{k}^{i}(f \mid s)=\left\{\begin{array}{lll}
U^{i}\left(f \mid s_{n}\right) & \text { if } & k=1, L+1,2 L+1, \ldots \\
U^{i}\left(f \mid s_{n+1 \bmod L}\right) & \text { if } & k=2, L+2,2 L+2, \ldots \\
& \vdots & \\
U^{i}\left(f \mid s_{n+L \bmod L}\right) & \text { if } & k=L, 2 L, 3 L, \ldots
\end{array}\right.
$$

Since by assumption $\lim _{k \rightarrow \infty} U_{k}^{i}(f \mid s)=\bar{u}_{i}$, it follows that $U^{i}\left(f \mid s_{l}\right)=\bar{u}_{i}$, for all $0 \leq l \leq L$. In particular, $U^{i}(f \mid s)=U^{i}\left(f \mid s_{n}\right)=\bar{u}_{i}$.

Finally, since $U^{i}(f \mid s)=(1-\delta) u_{i}(B(s))+\delta \bar{u}_{i}$ it follows that $u_{i}(B(s))=\bar{u}_{i}$.

As a consequence, we have Theorem 1, which yields a necessary condition on equilibrium strategies.

Theorem 1 If a strategy $f=\left(\left(S, s^{0}\right), T, B\right)$ is a finitely complex, symmetric, globally stable, semi-perfect equilibrium then $B(s)$ is a Nash equilibrium of $G$, for all $s \in S$.

Proof. Let $s \in S$ and $i \in N$. Then $B_{i}(s)$ is a static best reply to $B_{-i}(s)$, which establishes the theorem. The action $B_{i}(s)$ is a static best reply to $B_{-i}(s)$ is because otherwise player $i$ would deviate and play using a strategy that differs from $f_{i}$ only on the action played on state $s$.

More formally, assume that $B_{i}(s)$ is not a best reply to $B_{-i}(s)$. Let $a^{*}$ be a best reply in $G$ against $B_{-i}(s)$. In particular, $u_{i}\left(a^{*}, B_{-i}(s)\right)>u_{i}(B(s))=\bar{u}_{i}$. Define $\tilde{f}_{i}$ as follows: $\tilde{S}_{i}=S, \tilde{s}_{i}^{0}=s, \tilde{T}_{i}=T$ and

$$
\tilde{B}_{i}(\tilde{s})= \begin{cases}a^{*} & \text { if } \tilde{s}=s \\ B_{i}(\tilde{s}) & \text { otherwise. }\end{cases}
$$

It follows immediately that $\operatorname{comp}\left(\tilde{f}_{i}\right)=\operatorname{comp}\left(f_{i}\right)$. Also, letting $L$ denote the length of the cycle of $\left(\tilde{f}_{i}, f_{-i}\right) \mid s$, we obtain that $U_{i}\left(\left(\tilde{f}_{i}, f_{-i}\right) \mid s\right)>U_{i}(f \mid s)$, since

$$
\begin{aligned}
& U_{i}\left(\left(\tilde{f}_{i}, f_{-i}\right) \mid s\right)= \\
& (1-\delta) u_{i}\left(a^{*}, B_{-i}(s)\right)+\delta U_{i}\left(\left(\tilde{f}_{i}, f_{-i}\right) \mid T\left(s,\left(a^{*}, B_{-i}(s)\right)\right)=\right. \\
& (1-\delta) u_{i}\left(a^{*}, B_{-i}(s)\right)+\delta\left(\lambda u_{i}\left(a^{*}, B_{-i}(s)\right)+(1-\lambda) \bar{u}_{i}\right)> \\
& \bar{u}_{i}=U_{i}(f \mid s),
\end{aligned}
$$


where $\lambda=\frac{(1-\delta) \delta^{L-1}}{1-\delta^{L}}$. However, the above inequality is a contradiction to $f$ being a semi-perfect equilibrium.

Theorem 1 and Lemma 2 place strong restriction on the outcomes and payoffs that can arise in equilibrium. They imply that in every stage of a game in which players play in the way described in Theorem 1 we will observe a Nash equilibrium of the stage game. This result yields the following interpretation of Nash equilibria: In a given society, simple, symmetric, stable and minimally complex behavior is described by Nash equilibria.

Let $N(G)$ be the set of Nash equilibria payoffs in the stage game $G$, and let $E(G)$ be the set of finitely complex, symmetric, globally stable, semi-perfect equilibria payoffs in the repeated game. As an immediate consequence of Theorem 1, we obtain the following result.

Theorem $2 E(G)=N(G)$.

Theorem 2 reinforces the association expressed in Theorem 1 between Nash equilibria of a given stage game, and the simple, symmetric, stable, and minimally complex equilibria of its supergame: This association is exact for the payoff players receive in equilibrium.

Note that the association between Nash equilibrium outcomes of a given stage game, and the simple, symmetric, stable, and minimally complex equilibrium outcomes of its supergame is not exact. In fact, we may have an equilibrium outcome in the supergame consisting of oscillations between several different (but payoff equivalent) Nash equilibria.

Consider the following example of a coordination game. Let $n=2$ and $A_{i}=\left\{\alpha_{i}, \beta_{i}\right\}, i=1,2$. Preferences are lexicographic for both players. Let payoffs be given by

\begin{tabular}{|c|c|c|}
\hline $1 \backslash 2$ & $\alpha_{2}$ & $\beta_{2}$ \\
\hline$\alpha_{1}$ & 1,1 & 0,0 \\
\hline$\beta_{1}$ & 0,0 & 1,1 \\
\hline
\end{tabular}

Table 1: Payoff Function for Example 1

Consider the following strategy: $S=\left\{s^{0}, s^{1}\right\}, T\left(s^{0}, a\right)=s^{1}, T\left(s^{1}, a\right)=s^{0}$ for all $a$, and $B\left(s^{0}\right)=\left(\alpha_{1}, \alpha_{2}\right), B\left(s^{1}\right)=\left(\beta_{1}, \beta_{2}\right)$. This strategy is clearly finitely complex, symmetric, globally stable, and semi-perfect. 
However, one may argued that the above equilibrium outcome is unlikely: players would eventually agree to meet in just one location, because they would reduce the complexity of their behavior, while maintaining their payoff. In other words, that strategy is Pareto dominated by other strategy satisfying all the properties we are interested with. In general, we say a strategy $\sigma$ in a given game is a Pareto optimal equilibrium if it is Pareto optimal within the set of all equilibria (i.e., if it cannot be Pareto dominated by any other equilibrium). This definition applies both to the game $G$ and to the supergame of $G$, and for any equilibrium concept we may want to use.

Our next result characterizes the set of Pareto optimal, finitely complex, symmetric, globally stable, semi-perfect equilibria: They consist of repetitions of a Pareto optimal Nash equilibrium of the stage game. In this way, we obtain an exact association between Pareto optimal Nash equilibrium outcomes of a given game, and the Pareto optimal finitely complex, symmetric, globally stable, semi-perfect equilibrium outcomes of its supergame.

Theorem 3 A strategy $f$ is a Pareto optimal, finitely complex, symmetric, globally stable, semi-perfect equilibrium if and only if, $\operatorname{comp}\left(f_{i}\right)=1$, for all $i \in N$, and $B(s)$ is a Pareto optimal Nash equilibrium of $G$, for all $s \in S$.

Proof. (Sufficiency) "Playing a Pareto optimal Nash equilibria of $G$ always" constitutes a finitely complex, symmetric, globally stable, semi-perfect equilibrium. Since $N(G)=E(G)$, then this strategy is also Pareto optimal equilibrium in the repeated game.

(Necessity) By Theorem 1 and Lemma 2, $B(s)$ is a Nash equilibrium of $G$ and $u_{i}(B(s))=\bar{u}_{i}$. Therefore, $\operatorname{comp}\left(f_{i}\right)=1$, and $B\left(s^{0}\right)$ is a Pareto optimal equilibrium of $G$.

\section{Discussion}

We showed that in discounted repeated games strategies that are finitely complex, symmetric, globally stable, and semi-perfect must prescribe a Nash equilibria of the stage game in every period. Furthermore, a strategy is also Pareto optimal if and only if it prescribes some Pareto optimal Nash equilibrium of the stage game to be played in every period.

Our results provide an interpretation of Nash equilibrium points as the outcomes one should expect in situations where the properties we have as-

sumed seem relevant. As we mentioned in the introduction, social institutions 
provide such an example: if players interactions are guided by social institutions satisfying all those properties, behavior will be described by Nash equilibria. With this interpretation in mind, we can also use Theorem 3 to describe the Pareto optimal social institution in societies that can be appropriately described by a discounted repeated game.

When applied to the repeated prisoners' dilemma, the above results imply that there is a unique finitely complex, symmetric, globally stable, semiperfect equilibrium. In this equilibrium players play 'defect' in every period, and so any form of 'cooperative behavior' is impossible to obtain in a finitely complex, symmetric, globally stable and semi-perfect way. This result may provide a justification for the type of legal codes that we have, in which deviators are punished by the State for a typically limited amount of time. Given such a legal system, cooperation can be obtained in a way that the behavior of all private individual (i.e., everyone except the State) satisfies all the above properties: private individual need simply to play 'cooperative' in every period. A similar example is obtained by considering a group of gangsters in which those that are not loyal to the group are punished by its leader, or by someone in his close circle. In these examples, what is important is the existence of some player that does not care about complexity, and can therefore make credible the threat of punishments that will not be used in equilibrium.

Our interpretation of Nash equilibrium points as outcomes that can arise from repeated interaction of individuals through social institutions can provide an example in which the epistemic conditions for Nash equilibrium in normal form games established by Aumann and Brandenburger (1995) are very natural. One of their conditions is that the conjecture players make about the strategy of the others is commonly known. ${ }^{6}$ This assumptions has been criticized by Jacobsen (1996) on the grounds that it is not plausible to assume that one player knows what another player thinks. In our framework, any conjecture a player might form about other players can be interpreted as being part of the social institutions shaping players interaction. This interpretation renders Aumann and Brandenburger (1995, Theorem B)'s epistemic condition quite reasonable.

Theorem 1 states that the set of finitely complex, symmetric, globally stable, semi-perfect equilibrium payoffs in the repeated game equals the set of

\footnotetext{
${ }^{6}$ See Aumann and Brandenburger (1995, Theorem B). If there are only two players, then players conjectures need only to be mutually know, as their Theorem A shows.
} 
Nash equilibrium payoffs in the stage game. This result should be contrasted with those obtained for the set of Nash equilibrium payoffs in the repeated game: in particular, the Folk Theorem (see Aumann (1981), Fudenberg and Maskin (1986), and Rubinstein (1979)) shows, under mild conditions, that each individually rational payoff is a Nash equilibrium payoff, provided that the discount factor is sufficiently close to one. Since a similar conclusion does not hold for the set of finitely complex, symmetric, globally stable, semi-perfect equilibrium payoffs, we can interpret Theorem 2 as an "antiFolk-Theorem" result. Surprisingly, this result is obtained with simple, and reasonable conditions.

More important than the particular form of our results is the expression of the following economic idea: static Nash equilibrium is a reasonable concept to describe repeated strategic interactions guided by social institutions. It is thus important to study to what extent does this general result depends on the particular assumptions we made. In the appendix, we consider a weaker version of stability, and different notions of minimal complexity.

The notion of weak global stability that we study requires only that the continuation payoff after any state converges, and that the limit is independent of the state we start with. We show (see Theorem 4, and 5) that our results continue to hold when we replace global stability by weak global stability.

We also consider the effect of weakening semi-perfection to Nash equilibrium in the repeated game (but maintaining the requirement that players care both about payoffs and about complexity). In this case we show by an example that all of our results can fail. On the other hand, Theorem 5 shows that if we strengthen the requirements of Nash equilibrium by considering subgame perfect equilibrium (again maintaining the requirement that players care both about payoffs and about complexity), then all our results hold if we restrict attention to cycle states. Since we can interpret cycle states as longrun behavior, we can still associate social institutions with Nash equilibrium: if players use enduring social institutions, them their long-run behavior will be described by Nash equilibria.

Another variation that can be made is on the definition of complexity of a strategy. Banks and Sundaram (1990), in the same framework of ours but with only two players, consider a measure of complexity that takes into account not only the number of states of the smallest automaton implementing it, but also the number of transitions emanating from each state. They show, by using this complexity measure, that assuming finite complexity is enough 
to obtain the conclusion of Theorem 1. However, analogues of Theorem 2, and 3 are, in general, false. ${ }^{7}$

The idea that static Nash equilibria are reasonable descriptions of repeated strategic interactions is also present in the work of Green (1980), Sabourian (1990), and Al-Najjar and Smorodinsky (2001). These authors study repeated interactions in large societies, in which any player's payoffs depend on his choice, and an aggregate outcome. Under different assumptions, they show that in any subgame perfect equilibrium most players play static $\varepsilon$-best replies in every period, i.e., an approximate version of our Theorem 1 holds in their framework. Our results differ from these in two way: first, although expressing similar ideas, the framework they consider differs considerably from ours; and second, we obtain that, under the assumptions of Theorem 1, exact Nash equilibria will be played. ${ }^{8}$

As pointed out by Al-Najjar and Smorodinsky (2001), we may interpret our results as providing a justification of Markovian behavior. In our framework there is no exogenous state variable, and so in any (time-independent) Markovian strategy players play the same action profile in every period. Thus, Theorem 3 justifies Markovian strategies as a Pareto optimal way of obtaining finite complexity, symmetry, global stability, and semi-perfection. ${ }^{9}$

Markovian strategies are more appealing when there is an exogenous state variable, i.e., in the stochastic case. Hence, it would be interesting to have an analogue of Theorem 3 for stochastic games. If we allow the initial state of any automata to be randomly determined, then the main result of Carmona (2002a, chapter 4) shows that an analogue of Theorem 3 does not hold in general, non-Markovian strategies may be needed to obtain the above properties in a Pareto optimal way. However, if the initial state of each automaton is set deterministically (as is the case in the present paper), we

\footnotetext{
${ }^{7}$ As an example, consider the battle of the sexes game (see Fudenberg and Tirole (1991, page 19)) in which only pure actions are allowed, and in which players have lexicographic preferences in its supergame. Then, alternating between the two Nash equilibria of the stage game is a Pareto optimal equilibrium in the sense of Banks and Sundaram (1990), and the resulting supergame payoff does not equal the payoff of any stage game Nash equilibria.

${ }^{8}$ For an alternative interpretation of Nash equilibrium using large (i.e., non-atomic), but static, games see Barlo and Carmona (2002).

${ }^{9} \mathrm{~A}$ similar approach is taken in Carmona (2002a, Chapter 4), where monetary trading is rationalized as a Pareto efficient way to obtain the same properties. See also Carmona (2002b), in which the same result is established under weaker restrictions in a similar model.
} 
conjecture that an analogue of Theorem 3 will hold, at least for a large class of games.

\section{A On the Reciprocal of Theorem 1}

The following example shows that the condition in Theorem 1 is not sufficient. Let $n=2$ and $A_{i}=\left\{\alpha_{i}, \beta_{i}\right\}, i=1,2$. Preferences are lexicographic for both players. Let payoffs be given by

\begin{tabular}{|c|c|c|}
\hline $1 \backslash 2$ & $\alpha_{2}$ & $\beta_{2}$ \\
\hline$\alpha_{1}$ & 0,1 & $-1,-1$ \\
\hline$\beta_{1}$ & $0,-1$ & 0,1 \\
\hline
\end{tabular}

Table 2: Payoff Function for Example 2

The following strategy is such that in every stage players play a Nash equilibrium of the stage game, but which is not a semi-perfect equilibrium of the supergame: $S=\left\{s^{0}, s^{1}\right\}, T\left(s^{0}, a\right)=s^{1}, T\left(s^{1}, a\right)=s^{0}$ for all $a$, and $B\left(s^{0}\right)=\left(\alpha_{1}, \alpha_{2}\right), B\left(s^{1}\right)=\left(\beta_{1}, \beta_{2}\right)$. This strategy is clearly finitely complex, symmetric, globally stable, and semi-perfect.

\section{B Variations}

In this appendix, we study whether our results are robust to alternative definitions of stability and minimally complexity.

We can weaken the notion of global stability by requiring that the initial state should not have an effect in the long-run payoff level, without requiring that this payoff level be equal to the equilibrium level. The following definition formalizes this idea. ${ }^{10}$

Definition 4 Let $f \in \Sigma$ and let $\left(\bar{u}_{i}\right)_{i \in N}=\left(U^{i}(f)\right)_{i \in N}$. Then $f$ is weakly globally stable if for all $i \in N$, and $s, s^{\prime} \in S$,

$$
\lim _{k \rightarrow \infty} U_{k}^{i}(f \mid s)=\lim _{k \rightarrow \infty} U_{k}^{i}\left(f \mid s^{\prime}\right)
$$

\footnotetext{
${ }^{10}$ The definition of weak global stability was suggested to me by Narayana Kocherlakota.
} 
The notion of semi-perfection can also be weakened in a way suggested by Abreu and Rubinstein (1988).

Definition 5 (Abreu and Rubinstein (1988)) A strategy vector $f \in F$ is a $\succeq-N a s h$ equilibrium if for all $i \in N$, and $g_{i} \in F_{i}$,

$$
\left(U^{i}(f), \operatorname{comp}\left(f_{i}\right)\right) \succeq_{i}\left(U^{i}\left(g_{i}, f_{-i}\right), \operatorname{comp}\left(g_{i}\right)\right) .
$$

One can also define a subgame perfect equilibrium for any supergame in which preferences depend on both payoffs and complexity.

Definition 6 A strategy vector $f \in F$ is a $\succeq-$ subgame-perfect equilibrium if for all $i \in N, s \in S_{1} \times \cdots \times S_{n}$ and $g_{i} \in F_{i}$,

$$
\left(U^{i}(f \mid s), \operatorname{comp}\left(f_{i} \mid s\right)\right) \succeq_{i}\left(U^{i}\left(\left(g_{i}, f_{-i}\right) \mid\left(s_{i}^{g 0}, s_{-i}\right)\right), \operatorname{comp}\left(g_{i}\right)\right) .
$$

The next result says that weak global stability is all we need for our results.

Theorem 4 Theorems 1, 2, and 3 hold if we replace global stability with weak global stability.

Proof. It is enough to show that Lemma 2 still holds. This is clear: if we define $\bar{u}_{i}=\lim _{k \rightarrow \infty} U_{k}^{i}(f \mid s), s \in S$, then the same proof can be used.

However, our results are sensible to the notion of equilibrium used. If we use $\succeq-$ Nash equilibrium, then all our results may fail, as an example below shows. If we use $\succeq-$ subgame perfect equilibrium, then they all hold if we restrict attention to cycle states. Since we can interpret cycle states as longrun behavior, we can still associate social institutions with Nash equilibrium: if players use enduring social institutions, them their long-run behavior will be described by Nash equilibria.

For a given symmetric, and finitely complex strategy $f$ let $S_{c}(f)$ be the set of cycle states. This set is defined as follows: a symmetric, finite automaton induces a sequence of states $\left\{s_{k}\right\}_{k=1}^{\infty}$, where $s_{1}=s^{0}$, and $s_{k}=$ $T\left(s_{k-1}, B\left(s_{k-1}\right)\right)$ in a way that $t_{2}=\min \left\{m \in \mathbb{N}: s_{m+1}=s_{n}\right.$ for some $n \leq$ $m\}$ exists. Then $S_{c}(f)=\left\{s_{t_{1}}, \ldots, s_{t_{2}}\right\}$, where $t_{1} \leq t_{2}$ is such that $s_{t_{1}}=s_{t_{2}+1}$.

Lemma 3 If $f$ is a finitely complex, symmetric, and $\succeq-$ subgame perfect, then $T(s, a)$ belongs to $S_{c}(f)$ for all $s \in S_{c}(f)$, and $a \in A$. 
Proof. Let $s \in S_{c}(f)$, and $f$ be as above. We have that the set of states that will be used after $s$ is reached is $S_{c}(f)$. Hence, similarly to Lemma 1 , we conclude that the state space of $f \mid s$ is $S_{c}(f)$. Otherwise any player would remove any state outside of $S_{c}(f)$, which would reduce the complexity of $f \mid s$ while maintaining the payoff. Thus, $T(s, a) \in S_{c}(f)$.

Theorem 5 If a strategy $f=\left(\left(S, s^{0}\right), T, B\right)$ is a finitely complex, symmetric, weakly globally stable, $\succeq-$ subgame perfect equilibrium then $B(s)$ is a Nash equilibrium of $G$, for all $s \in S_{c}(f)$.

Proof. Assume that there exist $s \in S_{c}(f)$ that is not a Nash equilibrium of $G$. Let $i \in N$, and $a_{i}^{*}$ be such that $u_{i}\left(a_{i}^{*}, B_{-i}(s)\right)>u_{i}(B(s))$. Since $T\left(s,\left(a_{i}^{*}, B_{-i}(s)\right)\right) \in S_{c}(f)$ by Lemma 3 , and since, as one easily sees, the conclusion of Lemma 2 still holds, we can use the proof of Theorem 1 to show that $f$ is not $\succeq-$ subgame perfect.

Let $E_{c}(G)$ be the cycle payoff vectors supported by finitely complex, symmetric, weakly globally stable, $\succeq-$ subgame perfect equilibria. These payoff vectors can be thought of the payoff players will obtain in the long-run (note that these payoffs are well defined by an analogous version of Lemma 2.) As a consequence of Theorem 5 we have

Theorem $6 E_{c}(G)=N(G)$.

Finally, in order to obtain an analog to Theorem 3, we define the following notion of long-run Pareto optimality: an equilibrium $f \in F$ is Pareto optimal in the long run if it is not Pareto dominated in the long run by any other equilibrium. An equilibrium $f \in F$ Pareto dominates an equilibrium $g$ in the long run if for all $s \in S_{c}(f)$,

$$
\left(U_{i}(f \mid s), \operatorname{comp}\left(f_{i} \mid s\right) \succeq\left(U_{i}(g \mid s), \operatorname{comp}\left(g_{i} \mid s\right),\right.\right.
$$

for all $i \in N$, and

$$
\left(U_{j}(f \mid s), \operatorname{comp}\left(f_{j} \mid s\right) \succ\left(U_{j}(g \mid s), \operatorname{comp}\left(g_{j} \mid s\right),\right.\right.
$$

for at least one $j \in N$. We then obtain

Theorem 7 A strategy $f=\left(\left(S, s^{0}\right), T, B\right)$ is a Pareto optimal, finitely complex, symmetric, weakly globally stable, $\succeq-$ subgame perfect equilibrium if and only if, $\operatorname{comp}\left(f_{i} \mid s\right)=1$, for all $i \in N$, and $B(s)$ is a Pareto optimal Nash equilibrium of $G$, for all $s \in S_{c}(f)$. 
The following example shows that the conclusions of Theorems 1, 2, and 3 may fail when semi-perfection is weakened to $\succeq-$ Nash. Let $n=2$ and $A_{i}=\{C, D, M\}, i=1,2$. Preferences are lexicographic for both players, and players look first to the payoff. Let $\delta=6 / 10$, and stage game payoffs be given by

\begin{tabular}{|c|c|c|c|}
\hline $1 \backslash 2$ & $C$ & $D$ & $M$ \\
\hline$C$ & 6,6 & 0,7 & 0,0 \\
\hline$D$ & 7,0 & 1,1 & 0,0 \\
\hline$M$ & 0,0 & 0,0 & 3,3 \\
\hline
\end{tabular}

Table 3: Payoff Function for Example 3

Consider the following strategy $f$ : the state space is $S=\{D, C\}$, and $s^{0}=D$; the transition function is defined by

$$
T(D, a)= \begin{cases}C & \text { if } a=(D, D), \\ D & \text { otherwise }\end{cases}
$$

and

$$
T(C, a)= \begin{cases}C & \text { if } a=(C, C), \\ D & \text { otherwise }\end{cases}
$$

the behavior function is $B(D)=(D, D)$, and $B(C)=(C, C)$. This strategy is obviously symmetric, globally stable, and has finite complexity. It is easy to show that $U_{i}(f)>U_{i}\left(g_{i}, f_{-i}\right)$ for all $i \in N$, and $g_{i} \in \Sigma_{i}$. Hence, it follows that $f$ is a $\succeq-$ Nash equilibrium of the repeated game.

However, we have that $B(C)=(C, C)$ is not a Nash equilibrium of the stage game; hence the conclusion of Theorem 1 fails. The set of Nash equilibria payoffs in the stage game is $N(G)=\{(1,1),(3,3)\}$, but one sees that $U_{1}(f)=U_{2}(f)=1+5 \delta>3$. Thus, the conclusion of Theorem 2 fails. Finally, the last inequality also shows that the strategy that consists of playing the Pareto optimal Nash equilibrium $(M, M)$ of the stage game forever is not Pareto optimal in the repeated game.

Also, we can use the above game to give an example to show that we can not extend the conclusions of Theorems 5, 6, and 7 to all states. Consider the following strategy $f$ : the state space is $S=\left\{s^{0}, s^{1}, s^{2}\right\}$; the transition function is

$$
T\left(s^{0}, a\right)= \begin{cases}s^{1} & \text { if } a=(C, C), \\ s^{2} & \text { otherwise }\end{cases}
$$


$T\left(s^{1}, a\right)=s^{2}$, and $T\left(s^{2}, a\right)=s^{2}$ for all $a$; the behavior function is $B\left(s^{0}\right)=$ $(C, C), B\left(s^{1}\right)=(M, M)$, and $B\left(s^{2}\right)=(D, D)$. This strategy is obviously symmetric, globally stable, and has finite complexity. It is easy to show that $U_{i}\left(f \mid s^{l}\right)>U_{i}\left(g_{i}, f_{-i} \mid\left(s_{i}^{0 g}, s_{-i}^{l}\right)\right.$ for all $i \in N, s^{l}=s^{0}, s^{1}, s^{2}$, and $g_{i} \in \Sigma_{i}$. Hence, it follows that $f$ is a $\succeq$-subgame perfect equilibrium of the repeated game.

However, we have that $B\left(s^{0}\right)=(C, C)$ is not a Nash equilibrium of the stage game; hence the conclusion of Theorem 1 fails. The set of Nash equilibria payoffs in the stage game is $N(G)=\{(1,1),(3,3)\}$, but one sees that $U_{1}(f)=U_{2}(f)=6(1-\delta)+3 \delta(1-\delta)+\delta^{2}>3$. Thus, the conclusion of Theorem 2 fails. Finally, the last inequality also shows that the strategy that consists of playing the Pareto optimal Nash equilibrium $(M, M)$ of the stage game forever is not Pareto optimal in the repeated game.

\section{References}

Abreu, D., And A. Rubinstein (1988): "The Structure of Nash Equilibrium in Repeated Games with Finite Automata," Econometrica, 56, 1259-1281.

Al-NajJar, N., And R. Smorodinsky (2001): "Large Nonanonymous Repeated Games," Games and Economic Behavior, 37, 26-39.

Aumann, R. (1981): "Survey of Repeated Games," in Essays in Game Theory and Mathematical Economics in Honor of Oskar Morgenstern. Bibliographisches Institut, Mannheim.

Aumann, R., and A. Brandenburger (1995): "Epistemic Conditions for Nash Equilibrium," Econometrica, 63, 1161-1180.

Banks, J., and R. Sundaram (1990): "Repeated Games, Finite Automata, and Complexity," Games and Economic Behavior, 2, 97-117.

Barlo, M., and G. Carmona (2002): "Strategic Behavior in Non-Atomic Games: A Refinement of Nash Equilibrium," mimeo, University of Minnesota, and Universidade Nova de Lisboa.

Carmona, G. (2002a): "Essays on the Theory of Social Institutions, with Special Emphasis on Monetary Trading," Ph.D. thesis, University of Minnesota. 
(2002b): "Monetary Trading: An Optimal Exchange System," Working Paper 420, Universidade Nova de Lisboa.

- (2002c): "On the Notion of Social Institutions," Working Paper 421, Universidade Nova de Lisboa.

FudenberG, D., And E. Maskin (1986): "The Folk Theorem in Repeated Games with Discounting and Incomplete Information," Econometrica, 54, $533-554$.

Fudenberg, D., And J. Tirole (1991): Game Theory. The MiT Press, Cambridge, MA.

Green, E. (1980): "Non-Cooperative Price Taking in Large Dynamic Markets," Journal of Economic Theory, 22, 155-182.

Hopcroft, J., And J. Ullman (1979): Introduction to Automata Theory, Language, and Computation. Addison-Wesley, New York.

Jacobsen, H. (1996): "On the Foundations of Nash Equilibrium," Economics and Philosophy, 12, 67-88.

Kalai, E. (1990): "Bounded Rationality and Strategic Complexity in Repeated Games," in Game Theory and Applications, ed. by A. N. Tatsuro Ichiishi, and Y. Tauman. Academic Press, New York.

Kalai, E., And W. Stanford (1988): "Finite Rationality and Interpersonal Complexity in Repeated Games," Econometrica, 56, 397-410.

Kandori, M. (1992): "Social Norms and Community Enforcements," Review of Economic Studies, 59, 63-80.

Lipman, B., And S. SRivastava (1990): "Informational Requrements and Strategic Complexity in Repeated Games," Games and Economic Behavior, 2, 273-290.

McCulloch, W., And W. Pitts (1943): "A Logical Calculus for the Ideas Immanent in Nervous Activity," Bulletin of Mathematical Biophysics, 5, $115-133$.

Nash, J. (1950): "Non-Cooperative Games," Ph.D. thesis, Princeton University. 
Okuno-Fujiwara, M., and A. Postlewaite (1995): "Social Norms and Random Matching Games," Games and Economic Behavior, 9, 79-109.

Piccione, M. (1992): "Finite Automata Equilibria with Discounting," Journal of Economic Theory, 56, 180-193.

Roberts, A., And B. Holdren (1972): Theory of Social Processes. University of Iowa Press, Ames, Iowa.

Rubinstein, A. (1979): "Equilibrium in Supergames with the Overtaking Criterion," Journal of Economic Theory, 21, 1-9.

(1986): "Finite Automata Play the Repeated Prisoner's Dilemma," Journal of Economic Theory, 39, 83-96.

MA.

(1998): Modeling Bounded Rationality. The MIT Press, Cambridge,

Sabourian, H. (1990): "Anonymous Repeated Games with a Large Number of Players and Random Outcomes," Journal of Economic Theory, 51, 92-110.

Schotter, A. (1981): The Economic Theory of Social Institutions. Cambridge University Press, Cambridge.

van Damme, E. (1991): Perfection and Stability of Nash equilibrium. Springer Verlag, Berlin. 